\title{
Within-Digit Functional Parcellation of Brodmann Areas of the Human Primary Somatosensory Cortex Using Functional Magnetic Resonance Imaging at 7 Tesla
}

\author{
Rosa M. Sanchez-Panchuelo, ${ }^{1}$ Julien Besle, ${ }^{2}$ Alex Beckett, ${ }^{2}$ Richard Bowtell, ${ }^{1}$ Denis Schluppeck, ${ }^{2}$ and Susan Francis ${ }^{1}$ \\ ${ }^{1}$ Sir Peter Mansfield Magnetic Resonance Centre, School of Physics and Astronomy, and ${ }^{2}$ Visual Neuroscience Group, School of Psychology, University of \\ Nottingham, Nottingham, NG7 2RD, United Kingdom
}

\begin{abstract}
The primary somatosensory cortex ( $\mathrm{S} 1$ ) can be subdivided cytoarchitectonically into four distinct Brodmann areas (3a, 3b, 1, and 2), but these areas have never been successfully delineated in vivo in single human subjects. Here, we demonstrate the functional parcellation of four areas of S1 in individual human subjects based on high-resolution functional MRI measurements made at 7 T using vibrotactile stimulation. By stimulating four sites along the length of the index finger, we were able to identify and locate map reversals of the base to tip representation of the index finger in S1. We suggest that these reversals correspond to the areal borders between the mirrored representations in the four Brodmann areas, as predicted from electrophysiology measurements in nonhuman primates. In all subjects, maps were highly reproducible across scanning sessions and stable over weeks. In four of the six subjects scanned, four, mirrored, within-finger somatotopic maps defining the extent of the Brodmann areas could be directly observed on the cortical surface. In addition, by using multivariate classification analysis, the location of stimulation on the index finger (four distinct sites) could be decoded with a mean accuracy of $65 \%$ across subjects. Our measurements thus show that within-finger topography is present at the millimeter scale in the cortex and is highly reproducible. The ability to identify functional areas of S1 in vivo in individual subjects will provide a framework for investigating more complex aspects of tactile representation in S1.
\end{abstract}

\section{Introduction}

The primary somatosensory cortex (S1) is subdivided into four cytoarchitectonically distinct areas in the anterior-to-posterior plane: Brodmann areas 3a, 3b, 1, and 2 (Brodmann, 1909; Vogt and Vogt, 1919). Microelectrode recordings in nonhuman primates have revealed that each of these four areas contains a complete representation of the hand and fingers (Merzenich et al., 1978; Kaas et al., 1979; Nelson et al., 1980; Pons et al., 1985). Using functional magnetic resonance imaging (fMRI), several studies have resolved the mediolateral somatotopic organization of the finger representations in S1 in human subjects (Francis et al., 2000; Nelson and Chen, 2008; Schweizer et al., 2008; SanchezPanchuelo et al., 2010; Stringer et al., 2011). However, the reliable identification of distinct S1 areas in the anterior-to-posterior plane in vivo in individual human subjects has proven problematic. Most previous studies (Moore et al., 2000; Nelson and Chen,

Received May 24, 2012; revised Aug. 23, 2012; accepted Aug. 30, 2012.

Author contributions: R.M.S.-P., J.B., R.B., D.S., and S.F. designed research; R.M.S.-P., J.B., R.B., D.S., and S.F. performed research; A.B. contributed unpublished reagents/analytic tools; R.M.S.-P. and J.B. analyzed data; R.M.S.-P., J.B., R.B., D.S., and S.F. wrote the paper.

This work was supported by Biotechnology and Biological Sciences Research Council Grant BB/G008906/1 (D.S. and S.F.) and the Medical Research Council. D.S. is a Research Councils UK Academic Fellow. We thank Prof. Francis McGlone for his helpful comments on this manuscript and useful discussion.

Correspondence should be addressed to Rosa M. Sanchez-Panchuelo, Sir Peter Mansfield Magnetic Resonance Centre, School of Physics and Astronomy, University of Nottingham, University Park, Nottingham, NG7 2RD, UK. E-mail: rosa.panchuelo@nottingham.ac.uk.

DOI:10.1523/JNEUROSCI.2501-12.2012

Copyright $\odot 2012$ the authors $\quad 0270-6474 / 12 / 3215815-08 \$ 15.00 / 0$
2008; Overduin and Servos, 2008; Schweizer et al., 2008; Stringer et al., 2011) have attempted to assign functionally significant clusters of voxels to cytoarchitectonic areas based on anatomical criteria. However, the correspondence between cytoarchitectonic boundaries and macro-anatomical features identifiable from structural MR images is highly variable between subjects (Geyer et al., 1999) and might only be valid for group data in a probabilistic sense.

In addition to the well-established medial-to-lateral betweenfinger somatotopic organization of S1, electrophysiology studies in nonhuman primates have demonstrated an orthogonal anterior-to-posterior within-finger somatotopic organization corresponding to the representation of the proximal-to-distal (base-to-tip) surface of each finger (Paul et al., 1972; Merzenich et al., 1978; Kaas et al., 1979). For within-finger somatotopy, the mapping in each area of S1 mirrors the representation in the adjacent area (i.e., base-to-tip finger representation is posteriorto-anterior in area $3 \mathrm{~b}$ but anterior-to-posterior in area 1). Crucially, this layout results in map reversals of representations at the areal boundaries of areas 3a, 3b, 1, and 2 (Darian-Smith, 1982), providing a robust criterion to identify the different areas within S1. Only a limited number of fMRI studies have investigated within-finger somatotopy in human S1 (Blankenburg et al., 2003; Overduin and Servos, 2004, 2008; Schweisfurth et al., 2011), but to date, none have reliably identified the border of map reversals in individual subjects. Overduin and Servos (2008) did not show organized somatotopy using pneumatic stimulation of the thumb, index, and ring fingers. Blankenburg et al. (2003) re- 


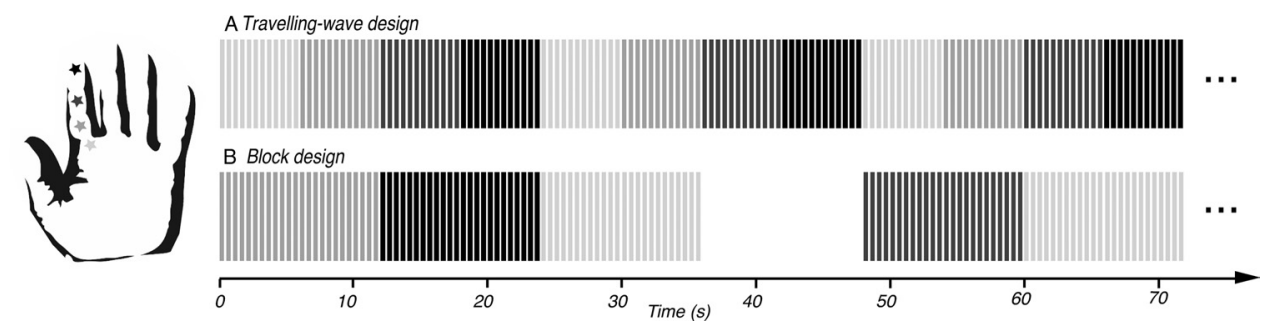

Figure 1. Experimental paradigms used to assess within-finger somatotopy. The asterisks in the hand legend represent locations of stimulation. $\boldsymbol{A}$, Traveling-wave paradigm: the index finger is stimulated sequentially either from the palm to the tip or in the reverse order. Shaded bars represent $6 \mathrm{~s}$ delivery of intermittent stimulation. $\boldsymbol{B}$, Block paradigm. Blocks of $12 \mathrm{~s}$ duration (consisting of 24 bursts of $0.4 \mathrm{~s}$ vibrotactile stimulation separated by $0.1 \mathrm{~s}$ gaps) were presented in a randomized order. Blocks consisting of $12 \mathrm{~s}$ rest periods were also presented.

ported an orderly anterior-to-posterior representation with mirrored patterns of clusters in areas $3 \mathrm{~b}$ and 1 at the group level but not in individual subjects. Schweisfurth et al. (2011) did not resolve within-finger somatotopy in the index finger but found a medial-to-lateral arrangement of the little finger base-to-tip representation of clusters in area $3 \mathrm{~b}$ in individual subjects.

Here, we exploit the improved BOLD contrast-to-noise ratio and high spatial resolution achievable at 7 Tesla to measure the topographic organization (base-to-tip) within the index-finger representation in $\mathrm{S} 1$ of individual subjects in response to punctate vibrotactile stimulation and thus to delineate distinct areas (analogous to Brodmann areas 3a, 3b, 1, and 2) based on functional maps alone.

\section{Materials and Methods}

Two methods were used to assess within-finger somatotopy: (1) a traveling-wave paradigm (Sanchez-Panchuelo et al., 2010) with standard Fourier-based analysis to form somatotopic maps of the within-finger index representation, and (2) a block design that allowed the use of a multivariate classification (or decoding) analysis for within-finger mapping. We used multivariate pattern analysis (MVPA) on the spatial pattern of responses across voxels in a given region of interest (ROI) to classify the responses to different stimulation locations, allowing the accuracy of discrimination between stimulation at different sites of the index finger to be assessed. The ROI was independently defined for each subject using data from a previous study (Besle et al., 2010) in which the five fingertips were sequentially stimulated using a traveling-wave design.

Subjects. Six subjects $(28.3 \pm 4.3$ years old, two females), experienced in fMRI experiments, participated in this study. Approval for the study was obtained from the University of Nottingham Ethics Committee, and all subjects gave full written consent. Each subject participated in two scan sessions: one session in which fMRI data was collected at $7 \mathrm{~T}$, and one session at $3 \mathrm{~T}$ to obtain a T1-weighted anatomical scan of the whole brain to be used for segmentation and cortical unfolding. Five of the subjects participated in an additional functional session to allow the reproducibility of the functional data to be evaluated.

Stimuli and paradigm. Vibrotactile stimuli were delivered to the index finger of the left hand using four independently controlled MRcompatible piezoelectric devices at the following four sites: the interdigital pad (the area of the palm beneath the finger) and the proximal (base), middle, and distal (tip) phalanx (Fig. 1). Each stimulator delivered a suprathreshold $(\sim 100 \mu \mathrm{m}) 150 \mathrm{~Hz}$ vibrotactile stimulus to $\sim 1 \mathrm{~mm}^{2}$ of the glabrous skin of each site (Dancer Design).

The somatosensory stimuli were presented in two fMRI paradigm designs within each session. The traveling-wave paradigm was designed so as to generate a traveling wave of activity across the somatotopic representation in S1 (Sanchez-Panchuelo et al., 2010). This involved applying stimuli sequentially to each site along the index finger in either a forward (palm to tip) or backward (tip to palm) order (Fig. 1A). Each site in turn was stimulated for $6 \mathrm{~s}$ by delivering twelve 0.4 -s blocks of continuous stimulation separated by $0.1 \mathrm{~s}$ (gaps were used to limit adaptation). A whole cycle of stimulation of all four sites thus took $24 \mathrm{~s}$. Each
fMRI run consisted of 10 cycles of stimulation, resulting in a total duration of $240 \mathrm{~s}$. In the block design experiment (Fig. $1 B$ ), each run comprised 20 stimulation blocks (four per site and four additional rest blocks). Each stimulation block was $12 \mathrm{~s}$ in duration, and stimuli were again delivered in $0.4 \mathrm{~s}$ bursts separated by $0.1 \mathrm{~s}$ gaps, so as to limit adaptation. The order in which each site was stimulated was pseudorandomized (i.e., four randomized sequences of palm, base, middle, tip, and rest stimulation blocks). Within a single scanning session, a total of four runs of the traveling-wave paradigm and six/seven runs of the block experiment were acquired. To control subjects' attentional state throughout the scan session, they performed a discrimination task in which they were required to make a visual contrast discrimination judgment on a fixation cross in a continuous two-interval alternative forcedchoice task. Subjects reported their response by pressing two buttons with their nonstimulated right hand, signaling in which interval the cross was darker. The contrast difference was continuously adjusted based on the subject's performance using a 3-down-1-up staircase procedure.

To evaluate the reproducibility of the within-finger somatotopic maps, five of the six subjects participated in a second fMRI scanning session in which traveling-wave data were again acquired.

Data acquisition. Data were acquired on a 7 T Philips Achieva system (Philips Medical Systems) using a volume transmit head coil and 32channel receive coil (Nova Medical). To minimize head motion, participants were stabilized with a customized MR-compatible vacuum pillow (B.u.W. Schmidt) and foam padding.

Functional data were obtained using multislice, single-shot gradient echo (GE), echo-planar imaging (EPI) with the following parameters: TE, $25 \mathrm{~ms}$; SENSE factor, 2; flip angle (FA), 75º TR, $2000 \mathrm{~ms}$. A total of 26 contiguous axial slices spanning the right $S 1$ were acquired with $1.25 \times$ $1.25 \times 1.3 \mathrm{~mm}^{3}$ resolution and a rectangular FOV of $192 \mathrm{~mm}$ in the anterior-posterior (AP) direction and $76 \mathrm{~mm}$ in the right-left phase encoding direction in conjunction with an outer-volume-suppression slab applied in the latter direction. An image-based shimming method to second order was used (Wilson et al., 2002; Poole and Bowtell, 2008), and the shim currents were fixed at the calculated values for all subsequent functional scans to ensure that geometric distortions were minimal and constant throughout the session (for details, see Sanchez-Panchuelo et al., 2010). This procedure leads to an approximately constant distortion as long as there is no significant head movement, as was the case for the experienced participants used in this study. A high-resolution $\mathrm{T}_{2}{ }^{*}$ weighted dataset $\left(0.3 \times 0.3 \times 0.65 \mathrm{~mm}^{3}\right.$ resolution) was also acquired with the same slice prescription as the functional data using a $3 \mathrm{D}$ fast, low-angle shot sequence (TE, $20 \mathrm{~ms}$; TR, $50 \mathrm{~ms}$; FA, $14^{\circ}$; acquisition time, $4 \mathrm{~min} 38 \mathrm{~s}$ ) to allow registration to the whole-head anatomical T1weighted images acquired at $3 \mathrm{~T}$. A high-resolution 3D MPRAGE dataset ( $1 \mathrm{~mm}$ isotropic resolution, linear phase encoding order; TE, $3.7 \mathrm{~ms}$; TR, $8.13 \mathrm{~ms}$; FA, $8^{\circ}$; TI, $960 \mathrm{~ms}$; acquisition time, $6 \mathrm{~min} 31 \mathrm{~s}$ ) was collected at $3 \mathrm{~T}$ for ease of segmentation before flattening, because images acquired at $3 \mathrm{~T}$ display less $\mathrm{B}_{1}$-inhomogeneity-related intensity variation compared with $7 \mathrm{~T}$ data.

Data processing. Analysis of functional imaging data was performed using a combination of custom-written software, mrTools (http://www. cns.nyu.edu/heegerlab) in MATLAB (MathWorks) and FSL (for FMRIB Software Library) (Smith et al., 2004). fMRI data were realigned within 

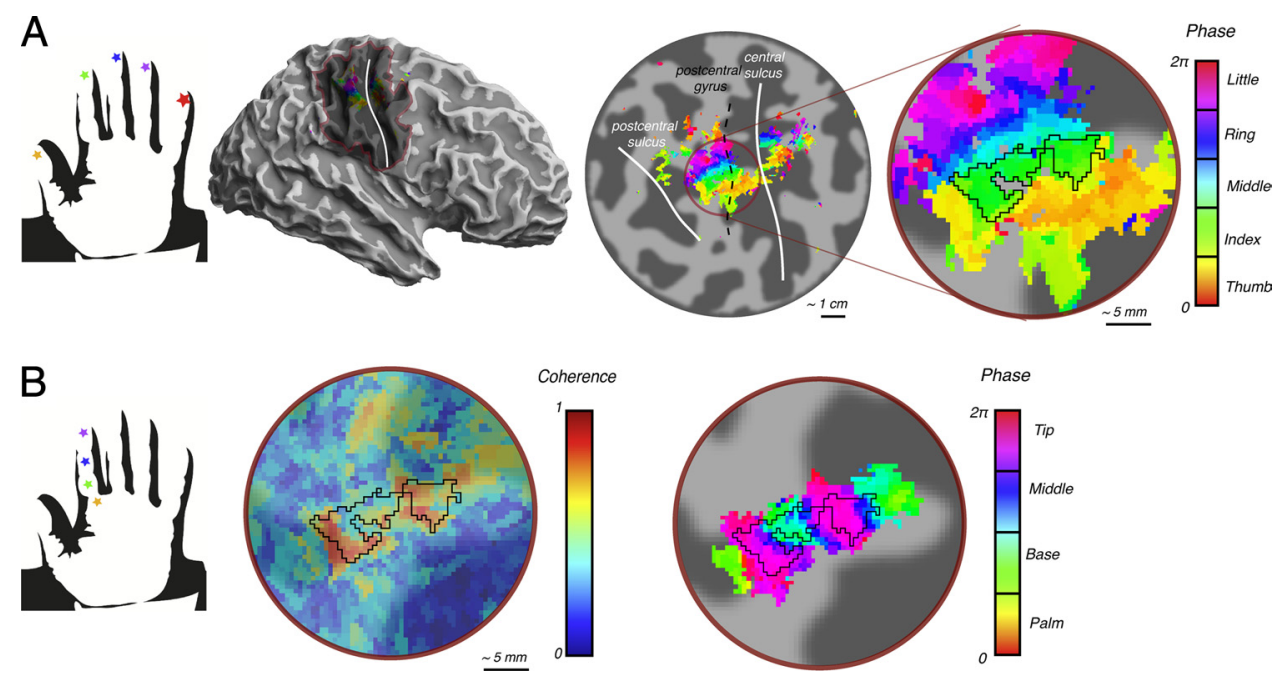

Figure 2. A, Results from a previous traveling-wave experiment (Besle et al., 2010) in which all fingertips of the left hand were stimulated (subject 1). Phase maps, thresholded at a coherence value of 0.25 , are displayed on an inflated 3D model of the right hemisphere cortical surface (left) and flattened cortical patch (right). Dark gray, Areas of negative curvature (sulci); light gray, areas of positive curvature (gyri); shaded area on the 3D model, location of the cortical flat patch. The orderly representation of the fingers is found in the posterior bank of the CS (white line) and the postcentral gyrus (dashed black line), corresponding to S1. The black line of the inset image represents the delineation of the index finger ROI, which consists of phase values encoded by the green color. $\boldsymbol{B}$, Results of the traveling-wave paradigm across sites of the index finger for subject 1. Coherence (left) shown with index fingertip ROl overlaid in black, and phase map (right) for the expanded ROI, thresholded at a coherence value of 0.25 , displayed over the same patch as inset in $\boldsymbol{A}$.

and between scans in a given fMRI session using a robust motioncorrection algorithm (Nestares and Heeger, 2000). To eliminate slow signal drift, the fMRI time series was then high-pass filtered $(0.01 \mathrm{~Hz}$ cutoff), and data were converted to percentage signal change for subsequent statistical analysis. The traveling-wave data were analyzed using a Fourier method (Engel et al., 1994), whereas the block data were analyzed using MVPA (Kamitani and Tong, 2005; Kamitani and Tong, 2006).

ROI definition. An independent ROI spanning the representation of the left-hand index finger in $\mathrm{S} 1$ was needed for the classification analysis and for assessment of the reproducibility of the traveling-wave mapping. For this purpose, the cortical representation of the index fingertip in S1 of each subject was derived from a previous scanning session, during which all five fingertips of the left hand were sequentially stimulated using a traveling-wave design (Besle et al., 2010). ROIs corresponding to each fingertip were defined in anatomical space by selecting clusters of contiguous voxels with a coherence value above 0.25 (corresponding to $p<$ 0.006 , uncorrected) and allocating phase values to five equally spaced bins, each of $2 \pi / 5$ width (between $2 \pi / 5$ and $4 \pi / 5$ for the index finger). As an example, the index fingertip ROI for subject 1 , which was defined from the traveling-wave phase map, can be seen in Figure $2 \mathrm{~A}$. To ensure that voxels responding to stimulation of more proximal portions of the index finger were also encompassed, the index fingertip ROI was expanded by adding in contiguous voxels displaying a coherence value $>0.25$ and lying within a distance of 4 voxels from the edge of the original ROI. This expanded index finger ROI was used for classification analysis and as the region studied in the Fourier analysis.

Traveling-wave within-finger Fourier data analysis. The time series from the proximal-to-distal and distal-to-proximal scans were combined as described by Sanchez-Panchuelo et al. (2010) to remove the effect of the hemodynamic delay in deriving the somatotopic maps. First, each scan was time shifted (advanced by 2 TRs) approximately to cancel any hemodynamic delay, and then the distal-to-proximal scans were time reversed before averaging of all the transformed scans. The average time series was analyzed voxel-by-voxel using standard Fourier-based analysis methods (Engel et al., 1994) yielding the phase, amplitude, and coherence to the best-fitting sinusoid at the stimulus repetition frequency. The resulting phase value from the combined time series reflects the timing of the proximal-to-distal stimulation and thus can be used to differentiate the location of the four stimulation sites.

The reproducibility of the phase maps across the two scan sessions was statistically assessed for each subject by computing the intersession phase difference for each voxel within the expanded index finger ROI. We tested whether the phase difference was randomly distributed around the circle $(2 \pi)$, against the alternative that the distribution of phase differences is unimodal and centered around 0 ( $V$ test; Durand and Greenwood, 1958) using the circular statistics toolbox for MATLAB (Berens, 2009).

Within-finger classification analysis of the block fMRI data. To determine whether the spatial pattern of the fMRI responses (rather than the voxelwise, univariate responses) contained information that would allow discrimination between stimulations on different sites on the index finger, we performed a multivariate classification analysis using the block data within the "expanded" index ROI for each subject. This analysis tests whether the stimulation site can be decoded from the spatial pattern of response in the expanded index ROI. The input patterns for classification are the voxels responses to the various stimulus blocks. For a given stimulus block, the vector of voxel responses was created by shifting the time series by $2-3$ TRs to correct for the hemodynamic delay and averaging the signal intensity in each voxel across all data points within the block. To train and test the classifiers, a "stimulation site decoder" was built using the methodology of Kamitani and Tong $(2005,2006)$. To test the accuracy of the classifier, a "leave one run out" cross-validation procedure (Kamitani and Tong, 2006) was used. The classifier was trained using the block fMRI data on all but one of the runs, and then its accuracy at classifying the patterns from the remaining runs was assessed. This procedure ensures the statistical independence of the test and training data, because they are drawn from separate runs. This process was repeated for each run in turn.

Anatomical registration, cortical segmentation, and flattening. Automated cortical segmentation of the anatomical 3 T MPRAGE data was performed using Freesurfer (http://surfer.nmr.mgh.harvard.edu/; Dale et al., 1999), and the mrFlatMesh algorithm (VISTA software, http:// white.stanford.edu/software/) was used to create flattened representations of the cortical regions surrounding the central sulcus (CS) and the postcentral gyrus. The high-resolution $\mathrm{T}_{2}{ }^{*}$-weighted data was linearly registered to the $\mathrm{T}_{1}$-weighted whole-head $3 \mathrm{~T}$ anatomical data. The reference EPI frame from the motion correction was linearly registered to the high-resolution $\mathrm{T}_{2}{ }^{*}$-weighted anatomical volume (Nestares and Heeger, 2000). Statistical results were rendered on a flattened patch of the cortical surface.

The inner (white matter border) and outer (pial) surfaces of the gray matter were identified and used to form nine surfaces that were equally 
spaced across the cortical thickness. The values of the statistical maps were interpolated across the cortical depth using nearest-neighbor interpolation. Values at a given coordinate (and thus cortical depth) in the original whole-head anatomical space were then displayed on the flattened patch by nearest-neighbor interpolation using the known direct correspondence between each point of the original inner/upper surface and each point of the flattened surface. The surface statistical maps were then restricted to the central cortical depth by finding the maximum intensity projection across the three central surfaces. This was done so as to limit contamination from the adjacent white matter and from the surrounding CSF and large surface vessels that could result from partial volume effects.

For surface rendering and ROI definition purposes, it is important that the gray/white matter segmentations of the MPRAGE images for each particular subject are in good agreement with the EPI data. Image-based shimming was used to minimize geometric distortions attributable to field inhomogeneity. However, when residual distortions remained, a nonlinear registration step between the functional EPI data and the highresolution $\mathrm{T}_{2}{ }^{*}$-weighted anatomy was also performed. For this step, we used the FSL nonlinear registration algorithm (FNIRT; Andersson et al., 2007) to warp the statistical maps into the structural $T_{2}{ }^{*}$-weighted data space. The results of the registration were compared with the anatomical landmarks of the $\mathrm{T}_{2}{ }^{*}$-weighted structural images. This nonlinear registration step was only performed when residual distortions were significant $(>1$ voxel). Nonlinear registration was required in 6 of the 11 datasets. Note that this registration was only used for display purposes and ROI definition: all analyses were performed on the original data (after motion correction), and only the resulting statistical maps were nonlinearly transformed for display on the cortical surface.

\section{Results}

\section{Traveling-wave within-finger Fourier data analysis}

The location of the index finger representation in S1 was obtained from a previous study (Besle et al., 2010) in which different fingertips were stimulated in a traveling-wave design, in the same six subjects that participated in the present study. Figure $2 \mathrm{~A}$ illustrates the procedure used to define the fingertip ROIs and shows the location of the fingertip somatotopic map in the posterior bank of the CS and on the postcentral gyrus in one example subject. Figure $2 B$ shows the results of the Fourier analysis of the within-index-finger traveling-wave paradigm in the same subject. Voxels with a high coherence value (i.e., high within-finger location specificity) are well localized within the index finger S1 ROI (for data from all subjects, see Fig. $3 A$ ), with stimulation of the palm, base, middle, and tip of the index finger consistently yielding location-specific responses (Fig. 3). The corresponding phase map, representing the preferred stimulation sites of the voxels on the index finger, shows an orderly pattern of phase variation along the index ROI, in a direction orthogonal to the mapping of the fingertips. Phase values alternate between magenta (tip) and blue/green (middle/base), with at least two reversals around the representation of the base (black and white dashed lines) and a single reversal around the phase value corresponding to the fingertip (white line), suggesting the existence of four mirrored within-finger maps in this subject.

The phase activation maps are shown for each individual subject in Figure $3 B$. Four of the six subjects scanned (subjects 1, 3, 4, and 6) exhibit an orderly pattern of phase variation along the index ROI in the AP direction with phase reversals consistent with mirrored representations in adjacent areas. The general pattern of reversals for these four subjects was compatible with the existence of four within-finger maps: the most anterior reversal around the fingertip (a), followed by a reversal around the base $(b)$, and then a second posterior reversal around the fingertip (c). Subject 6 was atypical in that this subject exhibited two reversals around the base of the index finger $(b, d)$. The two remaining subjects did not exhibit clear phase reversal patterns, although at least one phase progression from tip to base can be seen in subject 2 .

In general, phase values were weighted toward the fingertip stimulation, as shown by the greater number of magenta voxels (Fig. 3B) and histograms of phase values within the expanded index ROI (Fig. $3 C$ ). The mean \pm SD proportion of voxels within the ROI showing a preference for the tip stimulation was $47 \pm 9 \%$ (across subjects). The coherence values (Fig. 3A) were highest for voxels with a strongest response to stimulation of the tip with a mean across subjects of $0.37 \pm 0.06$ for the voxels with a preference for tip stimulation compared with $0.26 \pm 0.08$ for other voxels, reflecting the larger signal modulation produced by stimulation of the tip of the finger.

The within-finger traveling-wave paradigm was repeated in a second scan session in five subjects. Phase maps obtained in the two sessions were found to be highly reproducible, as shown by Figure 4 for both a subject exhibiting map reversals (subject 1 ) and a subject without clear reversals in their map (subject 2). The distribution of the intersession phase difference values in the expanded index finger ROI for each subject (Fig. $4 B$ ) was significantly non-uniform and distributed around 0 ( $V$ test, $V=$ $131.82,193.27,111.77,148.58$ and 118.08 for subjects $1,2,3,5$, and 6 , respectively, all $p<10^{-16}$ ). This illustrates that phase maps were highly reproducible betweens scanning sessions, even for those subjects who did not show mirrored phase patterns.

\section{Within-finger classification analysis of the block fMRI data}

Because not all subjects showed clear within-finger somatotopic maps, an MVPA was run using the spatial pattern of response in the index ROI to assess whether the within-finger stimulation site could be decoded based on the spatial pattern of activity. Figure 5 plots the ROI classification responses averaged across subjects. The classification accuracy was well above chance (25\%) for all subjects, ranging from 53 to $78 \%$ (mean accuracy across stimulation conditions for each subject: $65 \pm 8 \%$, average and SD across subjects). The classification performance for the tip trials was considerably higher than for the other stimulation conditions, as illustrated in Figure 5. The mean accuracy across subjects was $88 \%$ for the tip trials compared with 65,51 , and $58 \%$ for the palm, base, and middle stimulation sites, respectively.

\section{Discussion}

Using 7 T GE-BOLD fMRI, vibrotactile stimulation revealed location-specific activations in the index finger region of $\mathrm{S} 1$, in areas we suggest correspond to $3 \mathrm{a}, 3 \mathrm{~b}, 1$, and 2. Mirrored somatotopic representations in the AP direction, as predicted from monkey electrophysiology studies, were found in four of the six subjects scanned. The traveling-wave phase maps were highly reproducible across scan sessions, and the location of stimulation (palm/base/middle/tip) could be decoded using multivariate classification analysis, in all subjects.

\section{Comparison of functional parcellation with cytoarchitectonic areas}

Four subjects exhibited a pattern of map reversals consistent with site-specific activation of four adjacent mirrored maps within the index finger representation in S1. The most anterior reversal was always around the fingertip (a), followed posteriorly by a reversal around the base (b) and then another reversal around the tip (c). We suggest the four mirrored maps correspond to the four cytoarchitectonic areas first defined in humans (Brodmann, 1909; 
A Coherence map
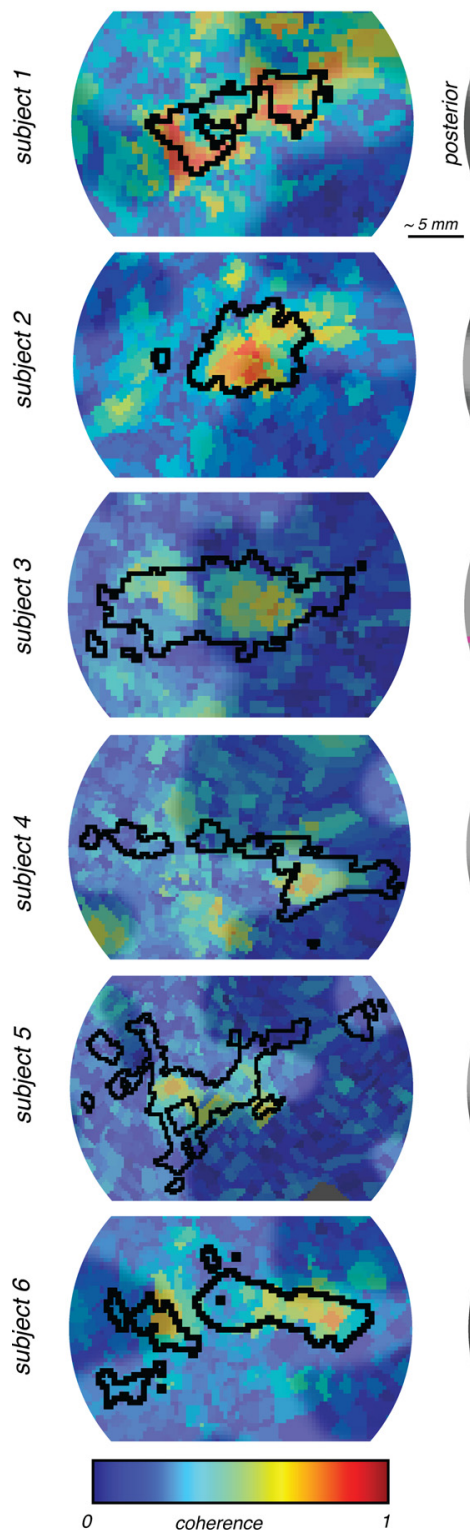

B Phase map
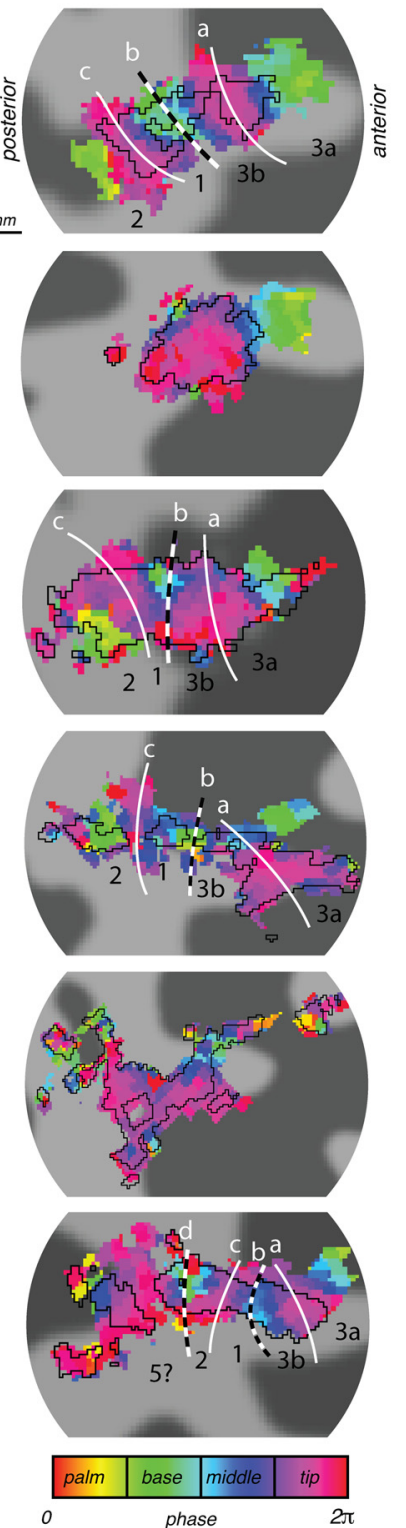

C Phase histogram
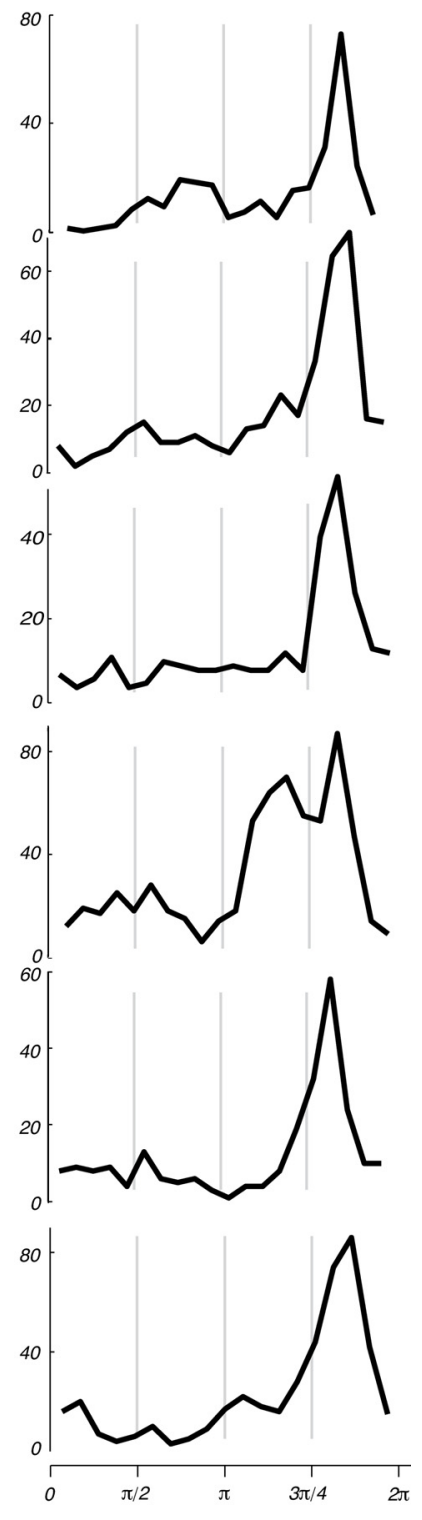

Figure 3. Within-finger traveling-wave data for all subjects. Each row shows data from one subject. $\boldsymbol{A}$, Coherence maps shown with index fingertip ROI overlaid in black. $\boldsymbol{B}$, Somatotopic (phase) within-finger maps. Displayed voxels correspond to the index finger ROI, expanded to encompass voxels showing preferential responses to proximal locations. White segments $(\boldsymbol{a}, \boldsymbol{c})$ and black and white dashed segments $(\boldsymbol{b}, \boldsymbol{d})$ emphasize phase reversals at the tip and base of the index finger, respectively. $\boldsymbol{C}$, Histogram of phase values for voxels displayed in the expanded index finger ROl shown in $\boldsymbol{B}$.

Vogt and Vogt, 1919) and later in macaque monkeys (Powell and Mountcastle, 1959). In monkeys, the locations of reversals of within-finger somatotopic maps identified from electrophysiology (Merzenich et al., 1978; Kaas et al., 1979; Nelson et al., 1980; Pons et al., 1985) have consistently been found to coincide with cytoarchitectonic borders, with the tip/base order being identical to that found in this study [most anterior fingertip reversal between $3 \mathrm{a}$ and $3 \mathrm{~b}$ (Krubitzer et al., 2004), base reversal between $3 \mathrm{~b}$ and 1 (Kaas et al., 1979), and posterior fingertip reversal between 1 and 2 (Kaas et al., 1979; Pons et al., 1985)].

Maps posterior to putative area 3 a were located in agreement with the location of cytoarchitectonic areas defined from postmortem measurements in humans (Geyer et al., 1999): the 3b/1 border was found in the CS, close to the crown of the postcentral gyrus (Blankenburg et al., 2003), and the 1/2 border was found on the postcentral gyrus. Subject 6 showed an additional posterior reversal around the base, suggesting an additional mirrored representation posterior to area 2 and could correspond to the human homolog of area 5 (Seelke et al., 2011) or to a second representation of the index in area 2, as shown in some macaque monkey individuals (Pons et al., 1985).

Two subjects did not show any evidence of mirrored maps, despite showing highly reproducible patterns of activation that were specific to stimulation of different sites on the finger. It is likely that these two subjects show a more convoluted pattern of maps, making it difficult to identify mirrored maps, which are expected to be aligned along a single rostrocaudal axis. Departure from strict somatotopy and convoluted maps are commonly observed in monkey electrophysiological studies (Merzenich et al., 1987). 

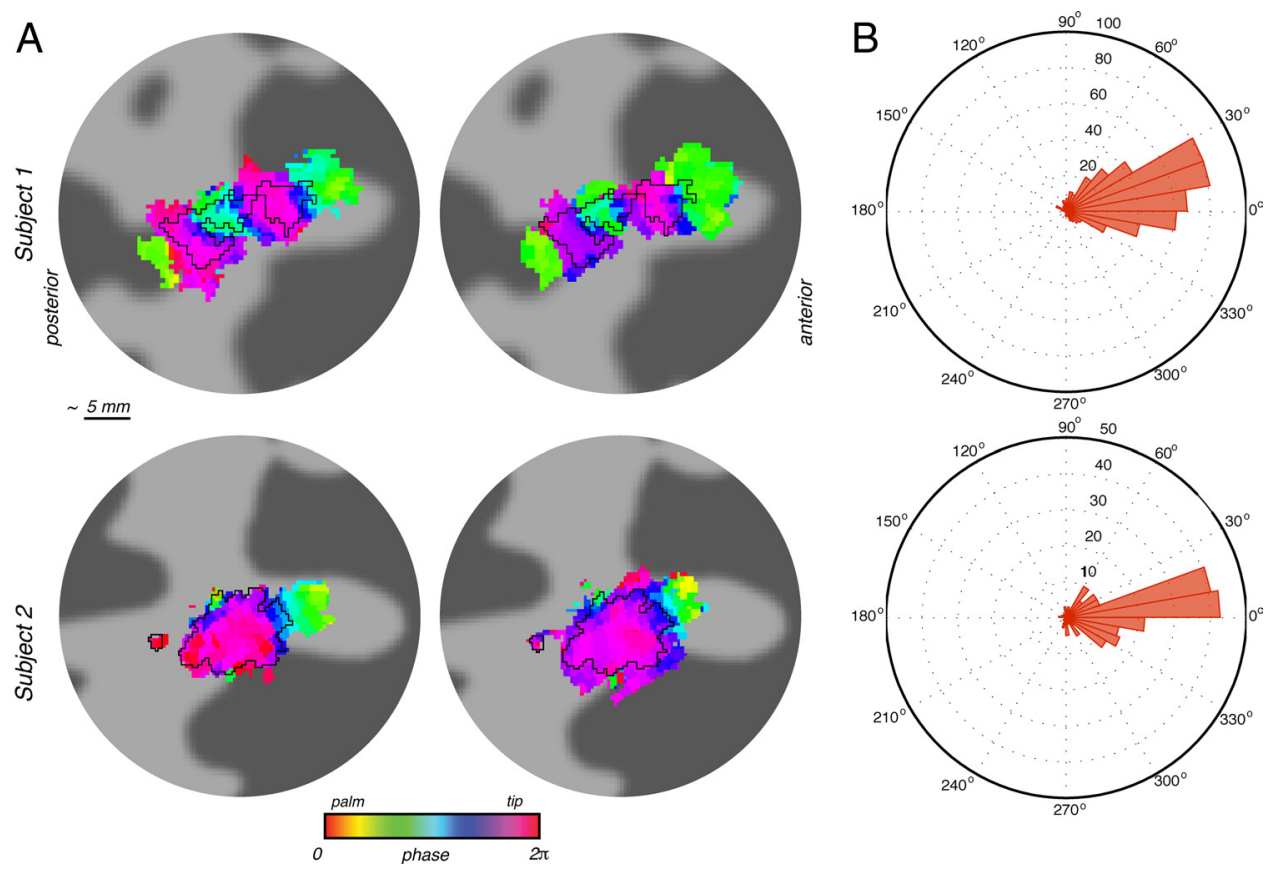

Figure 4. Reproducibility of within-finger somatotopic maps for a subject with mirror finger representations, subject 1 (top), and a subject with no mirror representations, subject 2 (bottom). $A$, Phase activation maps from data acquired in two separate scanning sessions. Black line shows index fingertip ROl with data shown for the expanded ROl. $\boldsymbol{B}$, Corresponding histograms of voxelwise phase differences between data acquired in two traveling-wave scanning sessions.

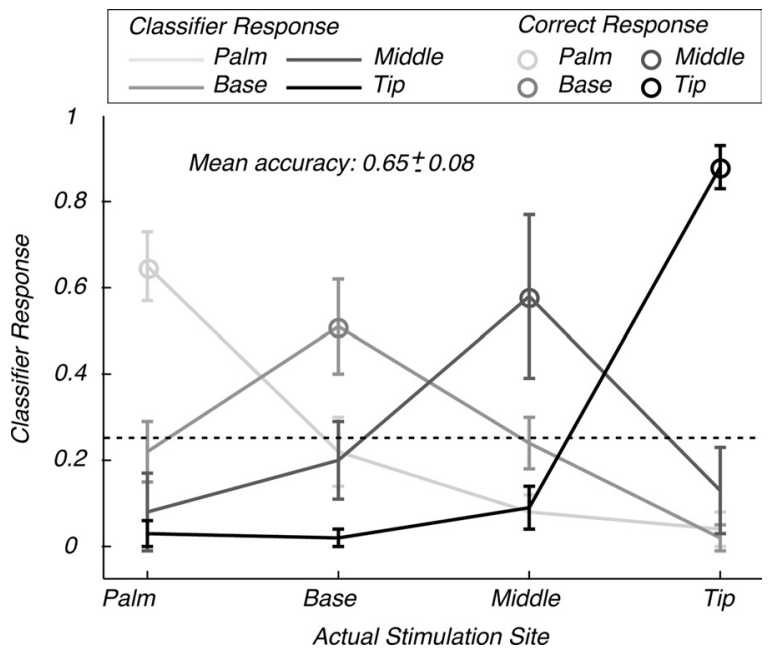

Figure 5. MVPA classifier response from the block-design data. Classification of test patterns from each stimulation site for the expanded index finger ROI (data averaged across subjects). Each curve represents the classifier responses to one stimulation site (palm, base, middle, and tip). Filled circles, Proportion of correct responses; error bars, SD. Dashed line, Chance level (25\%).

\section{Comparison with previous within-finger studies}

A recent 3 T fMRI human study using two sites of vibrotactile stimulation (Schweisfurth et al., 2011) showed evidence of singlesubject within-finger somatotopy in area $3 \mathrm{~b}$ for the little, but not the index, finger. This difference compared with our findings could result from the fact that the palm was used as the proximal location, whereas most of our subjects did not show a representation of the palm, or from the limited BOLD contrast-to-noise ratio available at $3 \mathrm{~T}$. At $7 \mathrm{~T}$, the increased BOLD contrast-tonoise ratio (Yacoub et al., 2001) and improved spatial specificity (Gati et al., 1997) may have contributed to improve the resolving power of our functional maps, allowing functional parcellation based on mirrored representations of the index finger in individual subjects. Furthermore, here we selectively sampled the middle cortical depths when displaying statistical maps on the flattened surface to reduce the spatial spread of the GE-BOLD response (Polimeni et al., 2010). Despite the small voxel size used here, spatial inaccuracy of the order of a voxel could potentially result in signals from extra-cortical veins being mapped onto the central layer (Koopmans et al., 2010). The activation on the anterior bank of the CS in Figure $2 A$ may originate from such inaccuracy. However, this activation is finger specific, implying that BOLD effects in the extracortical veins on the posterior bank of the CS show a high degree of finger specificity. Additional experimentation is needed to investigate this finding and the alternative hypotheses that the anterior signal represents finger-specific activation of the motor cortex or is attributable to displacement of signal from the central layer of the posterior bank of the CS (although this is unlikely given the high accuracy of image alignment achieved).

This study is the first to provide evidence for the existence of mirrored somatotopic maps beyond areas $3 \mathrm{~b}$ and 1 in humans. Previous fMRI studies contrasting stimulations at proximal and distal locations within a finger have only reported two mirrored maps (Blankenburg et al., 2003) or a single map (Schweisfurth et al., 2011). One reason might lie in the difficulty of using group analysis to uncover such fine-scale organization when averaging across subjects (which requires considerable smoothing). The variability of shape of the individual maps presented here clearly suggests that, to find reliable within-finger representations, mapping at the single-subject level is necessary.

Another possible reason for the lack of measured activation in areas 2 and 3 a in previous fMRI studies is that these areas respond less strongly than areas $3 \mathrm{~b}$ and 1 to weak cutaneous stimulation: more intense stimulation or movements of the limbs is needed to activate deep receptors (Pons et al., 1985; Krubitzer et al., 2004). Hence, cutaneous stimulations used in previous fMRI studies might have evoked weak responses that only reach statistical significance at $7 \mathrm{~T}$. 


\section{Comparison with previous fingertip somatotopy studies}

Previous fMRI studies of the $\mathrm{S} 1$ hand area involving stimulation of just the fingertips have often assigned the discrete activation clusters produced by stimulating a given fingertip to areas $3 \mathrm{~b}$ and 1 (Weibull et al., 2008; Stringer et al., 2011) and sometimes area 2 when three clusters were found (Ruben et al., 2006; Nelson and Chen, 2008). This interpretation conflicts both with our results and the existence of four mirrored maps. The map reversals at the fingertip in our activation maps (Fig. 3, magenta) suggest that stimulation of the fingertip alone would result in just two discrete clusters (putative borders of areas $3 \mathrm{a} / \mathrm{b}$ and $1 / 2$ ), rather than four clusters, because of the hemodynamic spatial blurring that prohibits the resolution of spatially separate BOLD responses in adjacent cortical representations of the fingertip, even at $7 \mathrm{~T}$. At lower magnetic fields, this effect would be more pronounced as a result of the broader hemodynamic point-spread function (Shmuel et al., 2007). Hence, we believe that activation clusters in previous fingertip studies could correspond to activation of all four subregions of $\mathrm{S} 1$ rather than just $3 \mathrm{~b}$ and 1 . Our data suggest that activation in areas 2 and 3 a should not be excluded on the ground that these two areas preferentially respond to deep receptor stimulation, because we do find at least four distinct maps, presumably corresponding to four distinct areas. The third posterior cluster of activation in the fingertip studies could stem from activation in area 5 (Seelke et al., 2011), additional representations in area 2 (Pons et al., 1985), or extra-vascular magnetic susceptibility effects attributable to large draining veins (Turner, 2002).

The data reported here indicate that cortical activation within the finger representation is weighted toward tip stimulation, in agreement with the findings of Overduin and Servos (2008). This is compatible with the higher density of mechanoreceptors found in the tip compared with the rest of the finger in humans (Johansson and Vallbo, 1979) and with the smaller receptive fields for the tip than the base in area $3 \mathrm{~b}$ of the macaque monkey (Pons et al., 1987), probably corresponding to a larger cortical magnification for the tip.

\section{Location-specific information can be decoded in all individual subjects}

The multivariate classification analysis showed that the location of the stimulation on the index finger could be decoded from the spatial pattern of BOLD activation within the index finger representation in S1, suggesting the presence of detailed internal maps of the finger, even for subjects who did not show any evidence of somatotopically organized maps. In a previous $3 \mathrm{~T}$ fMRI study (Beauchamp et al., 2009), MVPA was able to decode somatosensory stimuli widely separated on the surface (left and right hand and foot) as well as more closely located fingers of the same hand. Here, we have extended these findings to show that fMRI activation patterns in the index finger region of $\mathrm{S} 1$ contain information about different sites of stimulation along the finger.

\section{Conclusion}

7 T GE-fMRI allows delineation of four distinct Brodmann areas (3a, 3b, 1, and 2) in vivo in single human subjects from functional maps alone, a finding previously only possible using cytoarchitectonic criteria in postmortem brains. This parcellation was not seen in all subjects, most likely because of convoluted somatotopic maps in some subjects, although stimulation location information was present in the local spatial pattern of hemodynamic responses, as revealed by the MVPA. This functional parcellation, in conjunction with more complex experi- mental paradigms, will allow us to study the functional specialization of different areas of S1 in future studies.

\section{References}

Andersson JLR, Jenkinson M, Smith SM (2007) Non-linear registration, aka Spatial Normalization. Oxford: FMRIB Centre TR07JA2, http://www.fmrib.ox.ac.uk/ analysis/techrep/tr07ja2/tr07ja2.pdf.

Beauchamp MS, Laconte S, Yasar N (2009) Distributed representation of single touches in somatosensory and visual cortex. Hum Brain Mapp 30:3163-3171.

Berens P (2009) CircStat: a MATLAB toolbox for circular statistics. J Stat Soft 31:1-21.

Besle J, Sanchez-Panchuelo RM, Bowtell R, Francis S, Schluppeck D (2010) Event-related mapping of human primary somatosensory cortex at 7T. 16th Annual Meeting of the Organization for Human Brain Mapping, Barcelona, June 6-10, Abstract 1444

Blankenburg F, Ruben J, Meyer R, Schwiemann J, Villringer A (2003) Evidence for a rostral-to-caudal somatotopic organization in human primary somatosensory cortex with mirror-reversal in areas $3 \mathrm{~b}$ and 1 . Cereb Cortex 13:987-993.

Brodmann K (1909) Vergleichende Lokalisationslehre der Grosshirnrinde. Leipzig, Germany: Barth.

Dale AM, Fischl B, Sereno MI (1999) Cortical surface-based analysis. I. Segmentation and surface reconstruction. Neuroimage 9:179-194.

Darian-Smith I (1982) Touch in primates. Annu Rev Psychol 33:155-194.

Durand D, Greenwood JA (1958) Modifications of the Rayleigh test for uniformity in analysis of 2-dimensional orientation data. J Geol 66:229-238.

Engel SA, Rumelhart DE, Wandell BA, Lee AT, Glover GH, Chichilnisky EJ, Shadlen MN (1994) fMRI of human visual cortex. Nature 369:525.

Francis ST, Kelly EF, Bowtell R, Dunseath WJ, Folger SE, McGlone F (2000) FMRI of the responses to vibratory stimulation of digit tips. Neuroimage 11:188-202.

Gati JS, Menon RS, Ugurbil K, Rutt BK (1997) Experimental determination of the BOLD field strength dependence in vessels and tissue. Magn Res Med 38:296-302.

Geyer S, Schleicher A, Zilles K (1999) Areas 3a, 3b, and 1 of human primary somatosensory cortex 1. Microstructural organization and interindividual variability. Neuroimage 10:63-83.

Johansson RS, Vallbo AB (1979) Tactile sensibility in the human hand: relative and absolute densities of four types of mechanoreceptive units in glabrous skin. J Physiol 286:283-300.

Kaas JH, Nelson RJ, Sur M, Lin CS, Merzenich MM (1979) Multiple representations of the body within the primary somatosensory cortex of primates. Science 204:521-523.

Kamitani Y, Tong F (2005) Decoding the visual and subjective contents of the human brain. Nat Neurosci 8:679-685.

Kamitani Y, Tong F (2006) Decoding seen and attended motion directions from activity in the human visual cortex. Curr Biol 16:1096-1102.

Koopmans PJ, Barth M, Norris DG (2010) Layer-specific BOLD activation in human V1. Hum Brain Mapp 31:1297-1304.

Krubitzer L, Huffman KJ, Disbrow E, Recanzone G (2004) Organization of area $3 \mathrm{a}$ in macaque monkeys: contributions to the cortical phenotype. J Comp Neurol 471:97-111.

Merzenich MM, Kaas JH, Sur M, Lin CS (1978) Double representation of the body surface within cytoarchitectonic areas $3 \mathrm{~b}$ and 1 in "SI" in the owl monkey (Aotus trivirgatus). J Comp Neurol 181:41-73.

Merzenich MM, Nelson RJ, Kaas JH, Stryker MP, Jenkins WM, Zook JM, Cynader MS, Schoppmann A (1987) Variability in hand surface representations in areas $3 \mathrm{~b}$ and 1 in adult owl and squirrel monkeys. J Comp Neurol 258:281-296.

Moore CI, Stern CE, Corkin S, Fischl B, Gray AC, Rosen BR, Dale AM (2000) Segregation of somatosensory activation in the human rolandic cortex using fMRI. J Neurophysiol 84:558-569.

Nelson AJ, Chen R (2008) Digit somatotopy within cortical areas of the postcentral gyrus in humans. Cereb Cortex 18:2341-2351.

Nelson RJ, Sur M, Felleman DJ, Kaas JH (1980) Representations of the body surface in postcentral parietal cortex of Macaca fascicularis. J Comp Neurol 192:611-643.

Nestares O, Heeger DJ (2000) Robust multiresolution alignment of MRI brain volumes. Magn Reson Med 43:705-715.

Overduin SA, Servos P (2004) Distributed digit somatotopy in primary somatosensory cortex. Neuroimage 23:462-472. 
Overduin SA, Servos P (2008) Symmetric sensorimotor somatotopy. PLoS One 3:e1505.

Paul RL, Merzenich M, Goodman H (1972) Representation of slowly and rapidly adapting cutaneous mechanoreceptors of the hand in Brodmann's areas 3 and 1 of Macaca mulatta. Brain Res 36:229-249.

Polimeni JR, Fischl B, Greve DN, Wald LL (2010) Laminar analysis of $7 \mathrm{~T}$ BOLD using an imposed spatial activation pattern in human V1. Neuroimage 52:1334-1346.

Pons TP, Garraghty PE, Cusick CG, Kaas JH (1985) The somatotopic organization of area 2 in macaque monkeys. J Comp Neurol 241:445-466.

Pons TP, Wall JT, Garraghty PE, Cusick CG, Kaas JH (1987) Consistent features of the representation of the hand in area $3 \mathrm{~b}$ of macaque monkeys. Somatosens Res 4:309-331.

Poole M, Bowtell R (2008) Volume parcellation for improved dynamic shimming. MAGMA 21:31-40.

Powell TP, Mountcastle VB (1959) The cytoarchitecture of the postcentral gyrus of the monkey Macaca mulatta. Bull Johns Hopkins Hosp 105:108-131.

Ruben J, Krause T, Taskin B, Blankenburg F, Moosmann M, Villringer A (2006) Subarea-specific suppressive interaction in the BOLD responses to simultaneous finger stimulation in human primary somatosensory cortex: evidence for increasing rostral-to-caudal convergence. Cereb Cortex 16:819-826.

Sanchez-Panchuelo RM, Francis S, Bowtell R, Schluppeck D (2010) Mapping human somatosensory cortex in individual subjects with $7 \mathrm{~T}$ functional MRI. J Neurophysiol 103:2544-2556.

Schweisfurth MA, Schweizer R, Frahm J (2011) Functional MRI indicates consistent intra-digit topographic maps in the little but not the index finger within the human primary somatosensory cortex. Neuroimage 56:2138-2143.

Schweizer R, Voit D, Frahm J (2008) Finger representations in human pri- mary somatosensory cortex as revealed by high-resolution functional MRI of tactile stimulation. Neuroimage 42:28-35.

Seelke AM, Padberg JJ, Disbrow E, Purnell SM, Recanzone G, Krubitzer L (2012) Topographic Maps within Brodmann's area 5 of macaque monkeys. Cereb Cortex 22:1834-1850.

Shmuel A, Yacoub E, Chaimow D, Logothetis NK, Ugurbil K (2007) Spatiotemporal point-spread function of fMRI signal in human gray matter at 7 Tesla. Neuroimage 35:539-552.

Smith SM, Jenkinson M, Woolrich MW, Beckmann CF, Behrens TE, Johansen-Berg H, Bannister PR, De Luca M, Drobnjak I, Flitney DE, Niazy RK, Saunders J, Vickers J, Zhang Y, De Stefano N, Brady JM, Matthews PM (2004) Advances in functional and structural MR image analysis and implementation as FSL. Neuroimage 23 [Suppl 1]:S208-S219.

Stringer EA, Chen LM, Friedman RM, Gatenby C, Gore JC (2011) Differentiation of somatosensory cortices by high-resolution fMRI at 7 T. Neuroimage 54:1012-1020.

Turner R (2002) How much cortex can a vein drain? Downstream dilution of activation-related cerebral blood oxygenation changes. Neuroimage 16:1062-1067.

Vogt C, Vogt O (1919) Allgemeinere Ergebnisse unserer Hirnforschung. J Psychol Neurol 25:279-462.

Weibull A, Björkman A, Hall H, Ros én B, Lundborg G, Svensson J (2008) Optimizing the mapping of finger areas in primary somatosensory cortex using functional MRI. Magn Reson Imaging 26:1342-1351.

Wilson JL, Jenkinson M, de Araujo I, Kringelbach ML, Rolls ET, Jezzard P (2002) Fast, fully automated global and local magnetic field optimization for fMRI of the human brain. Neuroimage 17:967-976.

Yacoub E, Shmuel A, Pfeuffer J, Van De Moortele PF, Adriany G, Andersen P, Vaughan JT, Merkle H, Ugurbil K, Hu X (2001) Imaging brain function in humans at 7 Tesla. Magn Res Med 45:588-594. 